\title{
DISTRIBUTIVITY AND PERSPECTIVITY IN ORTHOMODULAR LATTICES
}

\author{
BY \\ SAMUEL S. HOLLAND, JR.(1)
}

1. Introduction. In this paper we prove for complete orthomodular lattices analogues of two results of continuous geometry.

$\operatorname{By}(a, b) P$, where $a, b$ are lattice elements von Neumann means that $a \wedge b=0$ and that for any $x$ in the lattice all six possible distributive laws for the triple $(a, b, x)$ hold [13, Part I, proof of Theorem 5.8]. The first result we consider is von Neumann's theorem: in a continuous geometry (not necessarily irreducible) if $\left(a_{\alpha}, b\right) P$ holds for all $\alpha$ in some indexing set $A$, then, setting $a=\wedge\left(a_{\alpha} ; \alpha \in A\right)$, $(a, b) P$ holds also [13, Part I, Theorem 5.8]. Kaplansky observed that this theorem is true in any complete complemented modular lattice, continuous or not [5, Lemma 36]. In this paper we prove that von Neumann's theorem holds in a complete orthomodular lattice, assuming neither modularity nor continuity (Theorem 1).

The second result we consider is Lemma 29 of [5]. In [5] Kaplansky proves: if $\left(a_{\alpha} ; \alpha \in A\right),\left(b_{\alpha} ; \alpha \in A\right)$ are two families of elements from a complete orthocomplemented modular lattice indexed by the same set $A$ such that $a_{\alpha}$ and $b_{\alpha}$ are perspective for every $\alpha$ in $A$ ("perspective" means they share a common complement) and such that $a_{\alpha} \vee b_{\alpha}$ is orthogonal to $a_{\beta} \vee b_{\beta}$ for $\alpha \neq \beta$, then $\bigvee a_{\alpha}$ and $\bigwedge b_{\alpha}$ are also perspective. We show (Theorem 3) in Kaplansky's result "orthocomplemented modular" can be replaced by "orthomodular," thus generalizing the result to a larger class of lattices. However to achieve the generalization we need to use the following form of the definition of the perspectivity of $a$ and $b$ : that there exists $x$ such that $a \vee x=b \vee x=a \vee b, a \wedge x=b \wedge x=0$. In a complemented modular lattice this is equivalent to requiring that $a$ and $b$ have a common complement [13, Part I, Theorem 3.1]. In an orthomodular lattice the two conditions are equivalent if and only if the lattice is modular (Theorem 2).

Orthomodular lattices derive their principal interest from the fact that they

Received by the editors April 15, 1963.

(1) These results were obtained while the author held a National Academy of SciencesNational Research Council Postdoctoral Associateship resident at the Air Force Cambridge Research Laboratories, Bedford, Mass., July 1960--July 1961. Lemma 6 and Theorems 2 and 3 were presented to the American Mathematical Society, October 28, 1961 under the title Analysis of weakly modular lattices. The paper was written while the author was supported in part by NSF grant GP-464. 
are a natural axiomatic prototype for the projection lattices of von Neumann algebras. The lattice of projections in a von Neumann algebra, and in particular the lattice of all projections on a Hilbert space, is always orthomodular. These projection lattices need not however be modular. This was proved for a real infinite dimensional normed linear space by Mackey [15, Theorem III-14 and Corollary 1 of Theorem III-6]. For Hilbert space (real or complex) the nonmodularity can be simply proved and we give the proof here. We make use of the fact that there exist in an infinite dimensional Hilbert space two closed subspaces $M$ and $N$ whose sum, $M+N$, is not closed $[3, \S 15]$. Select a vector $y$ in the closed linear span, $M \vee N$, of $M$ and $N$, but not in $M+N$, and let $P$ be the closed subspace spanned by $M$ and $y$. Since $M \leqq P$, modularity would imply that the subspaces $M \vee(N \wedge P)$ and $(M \vee N) \wedge P$ are equal. However this cannot be, since the vector $y$ belongs to the second one and not to the first. Consequently the lattice of all closed subspaces of (equivalently, the lattice of all projections on) an infinite dimensional Hilbert space is not modular.

Mackey has encountered orthomodular lattices in his study of the mathematical foundation of quantum mechanics [8, Chapter II, $\S \mathrm{B}]$. In his axiomatic development a basic role is played by certain observables, called questions, which form an orthocomplemented partially ordered set $Q$ in which the orthomodular law: $a \leqq b$ implies $b=a \vee\left(b^{\perp} \vee a\right)^{\perp}$ holds, but which need not be a lattice. The basic axiom for quantum mechanics is this: $Q$ is the lattice of all closed subspaces of an infinite dimensional separable Hilbert space [8, p. 113, Axiom VII]. Mackey expresses dissatisfaction with the ad hoc nature of this axiom, and points out the desirability of having a structure theory for the orthomodular partially ordered sets $Q$. A satisfactory structure theory for orthomodular lattices would allow one to reduce the axiom to the much weaker assumption that $Q$ is a lattice. Our results are part of such a theory. In $\$ 5$ we discuss some of the difficulties involved in devising a more complete theory along these same lines, and indicate possible directions for future study.

2. Notations and basic results. We denote the least upper bound of the elements $a, b$ of a lattice by $a \vee b$, and their greatest lower bound by $a \wedge b$. If $a$ is an element of the lattice $L$ with 0 and 1 , then an element $x$ of $L$ such that $a \vee x=1, a \wedge x=0$ is called a complement of $a$. A lattice Lcontaining 0 and 1 in which every element has a complement is called complemented, and is called orthocomplemented in case there is a one-to-one mapping, $a \rightarrow a^{\perp}$, of $L$ onto itself such that (1) $a^{\perp}$ is a complement of $a$, (2) $a \leqq b$ if and only if $a^{\perp} \geqq b^{\perp}$, and (3) $a^{\perp \perp}=a$. We call $a^{\perp}$ the orthocomplement of $a$. If $a \leqq b^{\perp}$ we say that $a$ and $b$ are orthogonal (the relation is easily shown to be symmetric) and write $a \perp b$. If $a \perp b$ we shall use $a \oplus b$ instead of $a \bigvee b$ to signify this fact, and shall also, to simplify some formulas, write $b-a$ for $b \wedge a^{\perp}$ in case $a \leqq b$. An orthomodular lattice is an orthocomplemented lattice which satisfies the axiom $a \leqq b \rightarrow b=a \vee\left(b \wedge a^{\downarrow}\right)$; 
in terms of the alternative notations this is simply $b=a \oplus(b-a)$. The lattice is called complete in case any subset of its elements has a least upper bound and a greatest lower bound. For a subset $\left(a_{\alpha} ; \alpha \in A\right)$ we shall denote these respectively by $\bigvee\left(a_{\alpha} ; \alpha \in A\right), \wedge\left(a_{\alpha} ; \alpha \in A\right)$. Often we shall abbreviate these to $\bigvee a_{\alpha}, \wedge a_{\alpha}$ respectively, and in case that $\left(a_{\alpha}\right)$ is an orthogonal family $\left(a_{\alpha} \perp a_{\beta}\right.$ if $\left.\alpha \neq \beta\right)$ shall often use $\oplus$ for $\vee$.

We will need certain results from [4] which we summarize here as Lemmas 1 through 5. First a cardinal definition: In an orthocomplemented lattice, $L$, the element $a$ is said to commute with $b$, written $a C b$, in case $a=(a \wedge b) \oplus\left(a \wedge b^{\perp}\right)$.

LEMMA 1. L is orthomodular if and only if $a C b$ implies $b C a$.

Assume now that $L$ is orthomodular. If, in Lemmas 2 and 3, the indexing set $A$ is infinite, assume also that $L$ is complete.

LEMMA 2. If $a C b$, then $a^{\perp} C b$ and $a^{\perp} C b^{\perp}$. If $a_{\alpha} C b$ for all $\alpha$ in $A$, then $\left(\bigvee a_{\alpha}\right) C b$ and $\left(\bigwedge a_{\alpha}\right) C b$.

LEMMA 3. If $a_{\alpha} C b$ for all $\alpha$ in $A$, then $\vee\left(b \wedge a_{\alpha}\right)=b \wedge\left(\vee a_{\alpha}\right)$ and $\bigwedge\left(b \vee a_{\alpha}\right)=b \vee\left(\bigwedge a_{\alpha}\right)$.

LEMMA 4. For any $x$ in $L, b(x)=(x-x \wedge a) \oplus(x \vee a)^{\perp}$ is a complement of $a$ and every complement of a can be written in this form for some $x$ in L. Moreover $b(x)=a^{\perp}$ if and only if $x C a$.

As in [4] we shall write $(a, b, c) T$ (read: $(a, b, c)$ is a distributive triple) to mean that all six distributive laws involving the three elements $a, b, c$ hold.

LEMMA 5. If one of $a, b, c$ commute with the other two, then $(a, b, c) T$.

We shall employ Lemma 5 frequently in our lattice-theoretic computations. It is of considerable usefulness as a unifying method in such computations.

Lemmas 1 through 5 are proved in $[4, \S 2]$. Unknown to us some were in print at the time [4] was written, and we should like to acknowledge here these earlier results. Lemma 1 was apparently first obtained by Nakamura [12]. We are indebted to Professor F. Maeda for this reference. Lemma 3 is essentially Lemma 4,3 in $[10, \S 4]$. There is further overlap with $[10, \S 4]$ and other results of $[4, \S 2]$ not quoted here concerning the center of an orthomodular lattice.

We should also like to call attention to [2] a paper submitted at about the same time as [4], in which D. J. Foulis, continuing earlier work on orthomodular lattices, proves a body of theorems centering about the commutativity relation which are very similar to those contained in $[4, \S 2]$. See also his announcement in the Amer. Math. Soc. Notices 8 (1961), Abstract 585-7.

3. The relation $(a, b) P$. We assume throughout this section that $L$ is a complete orthomodular lattice. Von Neumann's relation $(a, b) P$ between elements $a, b$ of $L$ 
was defined in the introduction; it means that $a \wedge b=0$ and that all six possible distributive laws hold for the triple $(a, b, x)$ for any choice of $x$ in $L$. Our first main theorem is this:

THEOREM 1. If $\left(a_{\alpha}, b\right) P$ for all $\alpha$ in an indexing set $A$, and $a=\bigvee\left(a_{\alpha} ; \alpha \in A\right)$, then also $(a, b) P$.

We will base our proof on the following lemma. First one piece of notation: if an element $x$ has the property that $y \leqq x, y C a$ implies $y=0$ we will write $x N C a$. This is a strong negation of commutativity asserting that 0 is the only subelement of $x$ which commutes with $a$.

Lemma 6. The following condition is equivalent to $(a, b) P:(*) a \perp b$ and whenever $\times N C a$, then $x C b$. In particular condition (*) is symmetric in $a$ and $b$.

Proof of Lemma 6. We establish first the implication $(a, b) P \rightarrow(*)$. One of the distributive laws contained in the assertion $(a, b) P$ is: $(a \vee x) \wedge b=x \wedge b$, and if we set $x=a^{\perp}$ in this formula it follows that $b=a^{\perp} \wedge b$. Hence $b \leqq a^{\perp}$, so that $a \perp b$. Now suppose that $x N C a$. It must then be true that $x \wedge a^{\perp}=0$, for otherwise $x \wedge a^{\perp}$ would be a nonzero subelement of $x$ orthogonal to, and thus commuting with, $a$. Then by the same distributive law just used we have:

$$
b \wedge x^{\perp}=b \wedge\left(a \vee x^{\perp}\right)=b \wedge\left(a^{\perp} \wedge x\right)^{\perp}=b \wedge 0^{\perp}=b \wedge 1=b
$$

whence $x \perp b$ so $x C b$. Thus $x$ NC $a$ implies $x C b$ and the condition (*) is satisfied.

Suppose now that (*) holds. The condition $a \wedge b=0$ follows from $a \perp b$, and it remains to prove that for arbitrary choice of $x$ in $L$, all six distributive laws for the triple $(a, b, x)$ hold. We observe first that from the single distributive law

$$
x=(x \vee a) \wedge(x \vee b) \text { for all } x \in L
$$

all five others can be derived. The two laws $a \wedge x=a \wedge(x \vee b)$ and $b \wedge x$ $=b \wedge(x \vee a)$ follow immediately by intersecting both sides of (1) with $a$ and $b$ respectively. Then spanning the first of these expressions with $b$ we obtain

$$
b \vee(a \wedge x)=b \vee(a \wedge(x \vee b))
$$

On the right side note that $b C a$ (since $b \perp a)$, and that $b C(x \vee b)$ (since $b \leqq x \vee b)$ so that we can distribute according to Lemma 5 . We obtain

$$
b \vee(a \wedge x)=(b \vee a) \wedge(b \vee x)
$$

which is the fourth distributive law, and the fifth follows by interchanging $a$ and $b$. The remaining relation is this: $x \wedge(a \vee b)=(x \wedge a) \vee(x \wedge b)$. To prove it we note first that $x \wedge a$ commutes with both $x$ and $b$ and so by Lemma 5

$$
(x \wedge a) \vee(x \wedge b)=((x \wedge a) \vee x) \wedge((x \wedge a) \vee b)=x \wedge((x \wedge a) \vee b)
$$


and then the proof is completed by applying the distributive law (2) just proved to the term $(x \wedge a) \vee b$. We obtain

$$
(x \wedge a) \vee(x \wedge b)=x \wedge(b \vee a) \wedge(b \vee x)=x \wedge(b \vee a)
$$

Thus to complete the proof of Lemma 6 it is enough to establish (1), and this we proceed to do. Given $x$, set $x_{1}=(x \wedge a) \oplus\left(x \wedge a^{\perp}\right), x_{2}=x-x_{1}$, and observe that since both $x \wedge a$ and $x \wedge a^{\perp}$ commute with $a$, so does $x_{1}$ by Lemma 2 . Observe further that $x_{2} N C a$. To show this it is enough to show that $x_{2} \wedge a$ $=: x_{2} \wedge a^{\perp}=0$, for if $c \leqq x_{2}$ and $c C a$, then

$$
c=(c \wedge a) \oplus\left(c \wedge a^{\perp}\right) \leqq\left(x_{2} \wedge a\right) \oplus\left(x_{2} \wedge a^{\perp}\right)=0
$$

so that $c=0$, and it follows that $x_{2} N C a$. Now

$$
x_{2} \wedge a=\left(x-x_{1}\right) \wedge a=x \wedge(x \wedge a)^{\perp} \wedge\left(x \wedge a^{\perp}\right)^{\perp} \wedge a \leqq(x \wedge a) \wedge(x \wedge a)^{\perp}=0
$$

and similarly $x_{2} \wedge a^{\perp}=0$, so that the relation $x_{2} N C a$ is established. Then, since we are assuming that (*) holds, $x_{2} C b$ obtains. Also $x_{2} C x_{1}$ holds (because $\left.x_{2} \perp x_{1}\right)$, so, by Lemma 2, $x_{2} C\left(b \vee x_{1}\right)$. Furthermore $x_{2} C(a \vee x)$, since $x_{2} \leqq a \vee x$, so that by Lemma 5 we conclude that $\left(a \vee x, b \vee x_{1}, x_{2}\right)$ form a distributive triple. We make use of this fact to decompose the right side of (1) as follows:

$$
\begin{aligned}
(a \vee x) \wedge(b \vee x) & =(a \vee x) \wedge\left(b \vee x_{1} \vee x_{2}\right) \\
& =\left((a \vee x) \wedge\left(b \vee x_{1}\right)\right) \vee\left((a \vee x) \wedge x_{2}\right) \\
& =\left((a \vee x) \wedge\left(b \vee x_{1}\right)\right) \vee x_{2} \\
& =\left(\left(a \vee x_{1} \vee x_{2}\right) \wedge\left(b \vee x_{1}\right)\right) \vee x_{2} \\
& =\left(\left(a \vee x_{1}\right) \wedge\left(b \vee x_{1}\right)\right) \vee\left(x_{2} \wedge\left(b \vee x_{1}\right)\right) \vee x_{2}
\end{aligned}
$$

(where in the last step we have applied Lemma 5 to the triple $\left(a \vee x_{1}, x_{2}, b \vee x_{1}\right)$ ). Continuing

$$
\begin{aligned}
(a \vee x) \wedge(b \vee x) & =\left(\left(a \vee x_{1}\right) \wedge\left(b \vee x_{1}\right)\right) \vee x_{2} \\
& =(a \wedge b) \vee x_{1} \vee x_{2}=(a \wedge b) \vee x
\end{aligned}
$$

where again the last step involves an application of Lemma 5 , this time to the triple $\left(a, b, x_{1}\right)$. This is permissible since $b$ commutes with both $a$ and $x_{1}$. This chain of equalities proves (1) and so concludes the proof of Lemma 6 .

Proof of Theorem 1. To complete the proof of the theorem we make use of condition (*) of Lemma 6 with $a$ and $b$ interchanged. The condition $a \perp b$ is immediate since if every $a_{\alpha} \leqq b^{\perp}$, then $a=\bigvee a_{\alpha} \leqq b^{\perp}$ as well. So it remains to show that $x N C b$ implies $x C a$. But if $x N C b$ then $x C a_{\alpha}$ for every $\alpha$ (because 
$\left(a_{\alpha}, b\right) P$ for every $\alpha$ is assumed), whence by Lemma 2, $x C a$. This completes the proof of Theorem 1 .

F. Maeda [9, p. 20] defines, in any lattice $L$ with $0, a \nabla b$ if $(a \vee x) \wedge b=x \wedge b$ for all $x$ in $L$, and says that $a$ and $b$ are separated if both $a \nabla b$ and $b \nabla a$ hold. Both these relations are obviously special cases of $(a, b) P$, so that if $(a, b) P$ obtains, then $a$ and $b$ are separated in the sense of F. Maeda. Examination of the proof of Lemma 6 reveals that in proving the implication $(a, b) P \rightarrow(*)$ we have used only the distributive law $(a \vee x) \wedge b=x \wedge b$. This is precisely F. Maeda's condition $a \nabla b$, and consequently $a \nabla b \rightarrow(*)$, or, in view of Lemma $6, a \nabla b$ implies $(a, b) P$. Thus the conditions $a \nabla b$ and $(a, b) P$ are in fact equivalent in an orthomodular lattice. Since $(a, b) P$ is symmetric in $a$ and $b$ it follows that the same is true of $a \nabla b$ and that this single condition implies that $a$ and $b$ are separated. Moreover from the equivalence of $(a, b) P$ and $a \nabla b$, and Anmerkung 2.2, Chapter I of [9] we have that: if $(a, b) P, a_{1} \leqq a, \mathrm{~b}_{1} \leqq b$, then also $\left(a_{1}, b_{1}\right) P$.

4. Perspectivity. In his development of the theory of continuous geometry, von Neumann makes constant use of the fact that two elements $a$ and $b$ of a complemented modular lattice share a common complement if and only if they share a common complement in their own span. That is to say, the two conditions on $a$ and $b$,

(1) there exists $x$ such that $a \vee x=b \vee x=1, a \wedge x=b \wedge x=0$;

(2) there exists $x$ such that $a \vee x=b \vee x=a \vee b, a \wedge x=b \wedge x=0$, are equivalent in the complemented modular case. This is no longer true in an orthomodular lattice as we show in our next theorem. We shall accordingly distinguish these concepts, calling (2) strong perspectivity, and calling (1) perspectivity. This latter term is in agreement with the original terminology of von Neumann [13, Part I, Definition 3.1].

THEOREM 2. In an orthomodular lattice L strong perspectivity implies perspectivity, but the converse holds if and only if $L$ is modular.

Proof. If $x$ effects the strong perspectivity between $a$ and $b$, then one checks easily that the element $y=x \oplus(a \vee b){ }^{\perp}$ is a complement of both $a$ and $b$, so that $a$ and $b$ are also perspective. Thus strong perspectivity implies perspectivity. Von Neumann proved that if $L$ is modular, then the converse holds [13, Part I, Lemma 3.1]. Finally suppose that the converse holds; that is, that perspectivity implies strong perspectivity. Let $a, c$ be elements of $L$ with $a \leqq c$. To prove $L$ modular we must show that for any $b$ in $L,(a \vee b) \wedge c=a \vee(b \wedge c)$. Set $p=(a \vee b) \wedge c, q=a \vee(b \wedge c)$. We claim: $r=(b-b \wedge q) \oplus(b \vee q)^{\perp}$ is a common complement of $p$ and $q$. It is a complement of $q$ by Lemma 4. Also, since $q \leqq p, r \vee p \geqq r \vee q=1$, so that $r \vee p=1$ and it remains only to check $r \wedge p=0$. Observe that $b \vee q=b \vee a \vee(b \wedge c)=b \vee a$ and that $b \wedge c \leqq b \wedge q$. Then: 


$$
\begin{aligned}
r \wedge p & =\left((b-b \wedge q) \oplus(b \vee q)^{\perp}\right) \wedge p \\
& =\left((b-b \wedge q) \oplus(a \vee b)^{\perp}\right) \wedge p \\
& =(b-b \wedge q) \wedge p \text { using Lemma } 5 \\
& \leqq b \wedge(b \wedge c)^{\perp} \wedge p=(b \wedge c) \wedge(b \wedge c)^{\perp}=0
\end{aligned}
$$

which establishes that $r$ is a common complement of $p$ and $q$. Thus $p$ and $q$ are perspective, and hence strongly perspective. But $q \leqq p$, so from $p \vee x=q \vee x$ $=p \vee q=p$ it follows that $x \leqq p$, and since also $x \wedge p=0$, that $x=0$. Whence $p=q$. Thus $L$ is modular and the theorem is proved.

We shall denote the relation " $a$ and $b$ are strongly perspective" by $a \sim b$. Our second main theorem is this:

THEOREM 3. Let $L$ be an orthomodular lattice. If $\left(a_{\alpha} ; \alpha \in A\right),\left(b_{\alpha} ; \alpha \in A\right)$ are two families of elements from $L$ with the same indexing set $A$ (if $A$ is infinite assume $L$ complete) such that $\left(a_{\alpha} \vee b_{\alpha}\right) \perp\left(a_{\beta} \vee b_{\beta}\right)$ if $\alpha \neq \beta$ and $a_{\alpha} \sim b_{\alpha}$ for all $\alpha$ in $A$, then $\bigoplus a_{a} \sim \bigoplus b_{\alpha}$.

The first lemma in the proof is the replacement in orthomodular lattices for Theorem 1.2 of Part I of [13].

LEMMA 7. If $(a \vee c) \perp(b \vee d)$, then $(a \oplus b) \wedge(c \oplus d)=(a \wedge c) \oplus(b \wedge d)$.

Proof. Following von Neumann let $e=a \vee c, f=b \vee d$. Since $a \perp f$ we have $a C f$ and similarly $c C f$. Then by Lemma $5,(a, c, f) T$, so that

$$
(a \wedge c) \vee f=(a \vee f) \wedge(c \vee f)
$$

and similarly

$$
(b \wedge d) \vee e=(b \vee e) \wedge(d \vee e)
$$

Since $a \perp b, a C b$, and since $a \leqq e, a C e$. Whence $a C(b \vee e)$. Since also $a C f$, we conclude again by Lemma 5 that $(a, f, b \vee e) T$, so that

$$
\begin{aligned}
(a \vee f) \wedge(b \vee e) & =(a \wedge(b \vee e)) \vee(f \wedge(b \vee e)) \\
& =a \vee(f \wedge(b \vee e)) \text { using } a \leqq e \\
& =a \vee((f \wedge b) \vee(f \wedge e)) \text { using } f C b \text { and } f C e
\end{aligned}
$$

(3) $\quad(a \vee f) \wedge(b \vee e)=a \vee b$

where the last step follows because $b \leqq f$ and $f \wedge e=0$. Similarly

$$
(c \vee f) \wedge(d \vee e)=c \vee d
$$

\section{Hence}




$$
\begin{aligned}
(a \vee b) \wedge(c \vee d)= & (a \vee f) \wedge(b \vee e) \wedge(c \vee f) \wedge(d \vee e) \text { by (3) and (4) } \\
= & (a \vee f) \wedge(c \vee f) \wedge(b \vee e) \wedge(d \vee e) \\
= & ((a \wedge c) \vee f) \wedge((b \wedge d) \vee e) \text { by (1) and (2) } \\
= & ((a \wedge c) \wedge((b \wedge d) \vee e)) \vee(f \wedge((b \wedge d) \vee e)) \text { using } \\
& (a \wedge c, f,(b \wedge d) \vee e) T \\
= & (a \wedge c) \vee(f \wedge((b \wedge d) \vee e)) \\
= & (a \wedge c) \vee(f \wedge b \wedge d) \vee(f \wedge e)=(a \wedge c) \vee(b \wedge d)
\end{aligned}
$$

where in the last step we have used $(f, b \wedge d, e) T$. This completes the proof of Lemma 7.

LEMMA 8. Let $A$ be a nonempty indexing set, and assume $L$ complete if $A$ is infinite. Let $\left(a_{\alpha} ; \alpha \in A\right),\left(b_{\alpha} ; \alpha \in A\right)$ be two families of elements from $L$ such that $\left(a_{\alpha} \vee b_{\alpha}\right) \perp\left(a_{\beta} \vee b_{\beta}\right)$ if $\alpha \neq \beta$. Then

$$
\left(\bigoplus a_{\alpha}\right) \wedge\left(\bigoplus b_{\alpha}\right)=\bigoplus\left(a_{\alpha} \wedge b_{\alpha}\right) .
$$

Proof. We shall prove the lemma by induction on the cardinality of the indexing set $A$.

Case 1. $A$ is finite. For card $(A)=1$, the lemma is obvious. For card $(A)$ $=2$, it is precisely Lemma 7 . For card $(A)=n>2$ we proceed by induction. To this end assume the truth of the lemma for card $(A)=n-1$. Set $x_{n}=\bigoplus\left(a_{i} ; 1 \leqq i \leqq n-1\right), a=\bigoplus\left(a_{i} ; 1 \leqq i \leqq n\right), y_{n}=\bigoplus\left(b_{i} ; 1 \leqq i \leqq n-1\right)$, $b=\bigoplus\left(b_{i} ; 1 \leqq i \leqq n\right)$, and note that $a=x_{n} \oplus a_{n}, b=y_{n} \oplus b_{n}$. By hypothesis $\left(a_{i} \vee b_{i}\right) \perp\left(a_{j} \vee b_{j}\right)$ for $i \neq j$, and it follows that $\left(x_{n} \vee y_{n}\right) \perp\left(a_{n} \vee b_{n}\right)$. Then applying Lemma 7 we obtain

$$
a \wedge b=\left(x_{n} \oplus a_{n}\right) \wedge\left(y_{n} \oplus b_{n}\right)=\left(x_{n} \wedge y_{n}\right) \oplus\left(a_{n} \wedge b_{n}\right) .
$$

The term $x_{n} \wedge y_{n}$ can be computed using the induction hypothesis:

$$
\begin{aligned}
x_{n} \wedge y_{n} & =\left[\bigoplus\left(a_{i} ; 1 \leqq i \leqq n-1\right)\right] \wedge\left[\oplus\left(b_{i} ; 1 \leqq i \leqq n-1\right)\right] \\
& =\bigoplus\left(a_{i} \wedge b_{i} ; 1 \leqq i \leqq n-1\right) .
\end{aligned}
$$

Substituting (2) in (1) we obtain $a \wedge b=\bigoplus\left(a_{i} \wedge b_{i} ; 1 \leqq i \leqq n\right)$, which is the desired result.

Case 2. $A$ is infinite. By Case 1 the lemma is valid for all finite $A$. We assume $A$ is infinite and prove the general validity of the lemma by transfinite induction by showing that its truth for all indexing sets $B$ with card $(B)<$ card $(A)$ implies its truth for $A$. Let $\Omega$ be the smallest ordinal corresponding to card $(A) . \Omega$ is a limit ordinal; i.e., does not have an immediate predecessor. The set $A$ may be replaced by the set of ordinals $(\alpha ; \alpha<\Omega)$. For $\alpha<\Omega$ set $x_{\alpha}=\bigoplus\left(a_{\beta} ; \beta \leqq \alpha\right)$, 
$r_{\alpha}=\bigoplus\left(a_{\beta} ; \alpha+1 \leqq \beta<\Omega\right)$ and set $a=\bigoplus\left(a_{\alpha} ; \alpha<\Omega\right)$ so that $x_{\alpha} \oplus r_{\alpha}$ $=a$. Similarly set $y_{\alpha}=\bigoplus\left(b_{\beta} ; \beta \leqq \alpha\right), \quad s_{\alpha}=\bigoplus\left(b_{\beta} ; \alpha+1 \leqq \beta<\Omega\right)$, and $b$ $=\bigoplus\left(b_{\alpha} ; \alpha<\Omega\right)$, so that $y_{\alpha} \oplus s_{\alpha}=b$. Set $c=\bigoplus\left(a_{\alpha} \wedge b_{\alpha} ; \alpha<\Omega\right)$. We wish to prove: $c=a \wedge b$. It is clear that $c \leqq a \wedge b$, so it is enough to show that $c \geqq a \wedge b$. By hypothesis $\left(a_{\alpha} \vee b_{\alpha}\right) \perp\left(a_{\beta} \vee b_{\beta}\right)$ for $\alpha \neq \beta$, and it follows that $\left(x_{\alpha} \vee y_{\alpha}\right) \perp\left(r_{\alpha} \vee s_{\alpha}\right)$ for all $\alpha<\Omega$. Then applying Lemma 7 we obtain

$$
a \wedge b=\left(x_{\alpha} \oplus r_{\alpha}\right) \wedge\left(y_{\alpha} \oplus s_{\alpha}\right)=\left(x_{\alpha} \wedge y_{\alpha}\right) \oplus\left(r_{\alpha} \wedge s_{\alpha}\right) \text {. }
$$

Since $\Omega$ is the least ordinal corresponding to card $(A)$, the set $(\beta ; \beta \leqq \alpha<\Omega)$ has cardinality strictly less than card $(A)$. Therefore by the induction hypothesis we may use the conclusion of the theorem to compute $x_{\alpha} \wedge y_{\alpha}$ :

(4) $x_{\alpha} \wedge y_{\alpha}=\left[\bigoplus\left(a_{\beta} ; \beta \leqq \alpha\right)\right] \wedge\left[\bigoplus\left(b_{\beta} ; \beta \leqq \alpha\right)\right]=\bigoplus\left(a_{\beta} \wedge b_{\beta} ; \beta \leqq \alpha\right) \leqq c$.

Substituting (4) into (3) we obtain

whence

$$
a \wedge b \leqq c \vee\left(r_{\alpha} \wedge s_{\alpha}\right) \quad \text { for all } \alpha<\Omega,
$$

$$
a \wedge b \leqq \wedge\left(c \vee\left(r_{\alpha} \wedge s_{\alpha}\right) ; \alpha<\Omega\right) .
$$

Observe now that $c C\left(r_{\alpha} \wedge s_{\alpha}\right)$ for all $\alpha<\Omega$. For always $a_{\beta} C\left(a_{\alpha} \wedge b_{\alpha}\right)$ for any $\alpha, \beta<\Omega$, so that an application of Lemma 2 tells us that $a_{\beta}$ commutes with $c=\bigoplus\left(a_{\alpha} \wedge b_{\alpha} ; \alpha<\Omega\right)$. Another application of Lemma 2 establishes that $c$ commutes with $r_{\alpha}=\bigoplus\left(a_{\beta} ; \alpha+1 \leqq \beta<\Omega\right)$. Similarly $c$ commutes with $s_{\alpha}$, and a third application of Lemma 2 yields $c C\left(r_{\alpha} \wedge s_{\alpha}\right)$ for all $\alpha<\Omega$. With this fact at hand we are able to apply Lemma 3 to (5) to obtain:

$$
a \wedge b \leqq c \vee\left(\wedge\left(r_{\alpha} \wedge s_{\alpha} ; \alpha<\Omega\right)\right)
$$

However

$$
\begin{aligned}
\wedge\left(r_{\alpha} \wedge s_{\alpha}\right) & =\wedge\left(a \wedge x_{\alpha}^{\perp} \wedge b \wedge y_{\alpha}^{\perp}\right) \\
& =a \wedge b \wedge\left(\wedge x_{\alpha}^{\perp}\right) \wedge\left(\wedge y_{\alpha}^{\perp}\right) \\
& =a \wedge b \wedge a^{\perp} \wedge b^{\perp}=0
\end{aligned}
$$

so that from (6) we conclude $a \wedge b \leqq c$, which proves Lemma 8 .

To prove Theorem 3 let $x$ be the element which produces the strong perspectivity between $a_{\alpha}$ and $b_{\alpha}$ and set $x=\bigoplus x_{\alpha}$. Then

$$
\left(\bigoplus a_{\alpha}\right) \vee x=\left(\bigoplus b_{\alpha}\right) \vee x=\left(\bigoplus a_{\alpha}\right) \vee\left(\bigoplus b_{\alpha}\right)
$$

is obvious. Since $a_{\alpha} \vee x_{\alpha}=a_{\alpha} \vee b_{\alpha}$, the hypothesis of the theorem implies that $\left(a_{\alpha} \vee x_{\alpha}\right) \perp\left(a_{\beta} \vee x_{\beta}\right)$ for $\alpha \neq \beta$, so that Lemma 8 applies. Therefore

$$
\left(\bigoplus a_{\alpha}\right) \wedge x=\left(\bigoplus a_{\alpha}\right) \wedge\left(\bigoplus x_{\alpha}\right)=\bigoplus\left(a_{\alpha} \wedge x_{\alpha}\right)=0
$$


and similarly $\left(\bigoplus b_{\alpha}\right) \wedge x=0$, so that $x$ effects the strong perspectivity between $\bigoplus a_{\alpha}$ and $\bigoplus b_{\alpha}$. This completes the proof of Theorem 3 .

COROLlaRY 1. If $a \wedge b^{\perp} \sim a^{\perp} \wedge b$, then $a \sim b$.

Proof. We note first that the "parallelogram law" holds for the strong perspectivity; i.e., $a \vee b-a \sim b-a \wedge b$ for all $a, b$ in the orthomodular lattice $L$. To prove this we must find an element $x$ which is a complement of both $a \vee b-a$ and $b-a \wedge b$ in their span, $(a \vee b-a) \vee(b-a \wedge b)$. A routine computation which we omit shows that the element $x=a-(a \wedge b) \oplus\left(a \wedge b^{\perp}\right)$ is effective for this purpose. Now we apply the parallelogram law to prove that $p=a-a \wedge b^{\perp}$ and $q=b-b \wedge a^{\perp}$ are strongly perspective. First note that $p^{\perp} \vee q=1$, and and $p^{\perp} \wedge q=0$. Then, by the parallelogram law,

$$
p=1-p^{\perp}=p^{\perp} \vee q-p^{\perp} \sim q-p^{\perp} \wedge q=q .
$$

The proof of Corollary 1 is completed by noting that if furthermore $a \wedge b^{\perp} \sim a^{\perp} \wedge b$, then by Theorem 3 we can add these strong perspectivities to get $a \sim b$.

In [1], A. Brown proves: "in any ring of operators the equivalence of $E$ and $F$ follows from that of the orthogonal projections $E \wedge(I-F)$ and $F \wedge(I-E)$." His proof is operator theoretic. Based on Corollary 1 to Theorem 3 we can give a simple lattice-theoretic proof of the same result. Set $A=E \wedge(I-F)=E \wedge F^{\perp}$, $B=F \wedge(I-E)=F \wedge E^{\perp}$ and note that if $W$ is the partial isometry taking $A$ on $B$, then $1 / 2\left(A+B-W-W^{*}\right)$ is a projection which implements a strong perspectivity between the orthogonal projections $A$ and $B$. Then by Corollary 1 it follows that $E$ and $F$ are strongly perspective. Let $X$ be the projection which effects this strong perspectivity; we have $E \vee X=F \vee X=E \vee F, E \wedge X=F \wedge X=0$. Denote equivalence in the ring of operators by $\sim$ and recall that this equivalence also satisfies the parallelogram law. We then establish the equivalence of $E$ and $F$ in the ring of operators as follows:

$$
E=E-E \wedge X \sim E \vee X-X=F \vee X-X \sim F-F \wedge X=F .
$$

Since the hypothesis " $E \wedge F^{\perp}$ and $E^{\perp} \wedge F$ are equivalent" is unaffected by substituting $E^{\perp}$ for $E$ and $F^{\perp}$ for $F$, it follows also that $E^{\perp} \sim F^{\perp}$, and so that $E$ is unitarily equivalent to $F$.

We conclude this section by deducing from Theorem 3 the analogue for complete orthomodular lattices of Theorem 2.1 in [13, Part III]. This theorem, due to von Neumann, says: if $a, b$ are elements of the continuous geometry $L$ then there exists $a^{\prime}, a^{\prime \prime}, b^{\prime}, b^{\prime \prime}$ such that $a^{\prime} \wedge a^{\prime \prime}=0, a^{\prime} \vee a^{\prime \prime}=a ; b^{\prime} \wedge b^{\prime \prime}=0, b^{\prime} \vee b^{\prime \prime}=b$; $a^{\prime} \sim b^{\prime}$ and $\left(a^{\prime \prime}, b^{\prime \prime}\right) P$. To generalize this result we must replace the condition: " $\left(a\right.$ ",$\left.b^{\prime \prime}\right) P$ '" by: "if $p \leqq a ", q \leqq b^{\prime \prime}$, and $p \sim q$, then $p=q=0$ ". These two conditions are equivalent in a continuous geometry, but are not equivalent in 
complete orthomodular lattices. We discuss this point further in the next section . Using the second condition, we can state our theorem as follows:

Corollary 2. Let $a, b$ be elements of the complete orthomodular lattice $L$. Then there exist $a^{\prime}, a^{\prime \prime}, b^{\prime}, b^{\prime \prime}$ such that

(1) $a=a^{\prime} \oplus a^{\prime \prime}, b=b^{\prime} \oplus b^{\prime \prime}$;

(2) $a^{\prime} \sim b^{\prime}$;

(3) if $p \leqq a^{\prime \prime}, q \leqq b^{\prime \prime}$ with $p \sim q$, then $p=q=0$.

Proof. Let $p=a-a \wedge b^{\perp}, q=b-a{ }^{\perp} \wedge b$. Then $p \leqq a, q \leqq b$, and a routine computation shows that $p^{\perp} \wedge q=p \wedge q^{\perp}=0$. Hence $p \sim q$ by Corollary 1. Set $r=a \wedge b^{\perp}=a-p$ and $s=a^{\perp} \wedge b=b-q$. Then $r \perp s$, $a=p \oplus r, b=q \oplus s$. Now examine $r$ and $s$ for nonzero strongly perspective subelements. If none exist, the proof concludes with $a^{\prime}=p, a^{\prime \prime}=r, b^{\prime}=q, b^{\prime \prime}=s$. Otherwise consider pairs $(x, y)$, with $0 \neq x \leqq r, 0 \neq y \leqq s, x \sim y$. Call a set $S$ of such pairs a $p$-set if $(x, y) \in S,(u, v) \in S \rightarrow x \perp u, y \perp v$ or $x=u, y=v$. The set $P$ of all $p$-sets is partially ordered by set theoretic inclusion, and is clearly inductive. Then Zorn's lemma assures us of the existence of a maximal element in $P$, say $S$. Setting $r_{1}=\bigoplus(x ;(x, y) \in S) s_{1}=\bigoplus(y ;(x, y) \in S)$, we have that $r_{1} \leqq r$, $s_{1} \leqq s$, and by Theorem 3, $r_{1} \sim s_{1}$. Since $S$ is maximal, $r-r_{1}$ and $s-s_{1}$ do not contain nonzero strongly perspective subelements. Finally set $a^{\prime}=p \oplus r_{1}$, $a^{\prime \prime}=r-r_{1}, b^{\prime}=q \oplus s_{1}, b^{\prime \prime}=s-s_{1}$ and observe that by Theorem 3 again we can add to get $a^{\prime} \sim b^{\prime}$. This completes the proof.

5. Concluding remarks. In this section we present a sample of other theorems of continuous geometry which unlike von Neumann's and Kaplansky's results mentioned in the introduction fail to generalize to orthomodular lattices. These results are collected into one Theorem, Theorem 4 below. They indicate the difficulties one must face in attempting to devise a more complete theory of orthomodular lattice using the methods created by von Neumann.

They may also serve a more constructive purpose. As noted in Theorem 4 these results are valid in the projection lattice (modular or not) of any von Neumann algebra. Hence their failure to generalize to orthomodular lattices is not caused simply by the lack of the modular law. It rather reflects the extent to which orthomodular lattices overgeneralize projection lattices. The question then naturally arises: Is there a "natural" sub-class of orthomodular lattices which share the same lattice-theoretic properties as the projection lattices of von Neumann algebras? An investigation along these lines was probably in Kaplansky's mind when he suggested a "more general lattice project" than von Neumann's continuous geometry [6, Preface]. The list of conditions in Theorem 4 should be helpful as a guide in this project.

If $L$ is complemented modular interpret the relation $a \perp b$ in (3), (5) and (6) of 
Theorem 4 below as $a \wedge b=0$. The condition $(a, b, c) \perp$ of (7) means that $(x \vee y) \perp z$ for any permutation $x, y, z$ of the elements $a, b, c$.

THEOREM 4. Each of the following statements is true if $L$ is either a complete complemented modular lattice or the lattice of projections of a von Neumann algebra. None of the statements is true in general if $L$ is only complete orthomodular.

(1) For $a \in L$, define $a^{\nabla}=\bigvee(x ;(x, a) P)$. Then $a^{\nabla}$ is in the center of $L$.

(2) For $a, b \in L,(a, b) P$ is equivalent to: $a_{1} \leqq a, b_{1} \leqq b, a_{1} \sim b_{1}$ imply $a_{1}=b_{1}=0$.

(3) For $a, b \in L,(a, b) P$ is equivalent to: $a \perp b$ and $x \wedge(a \oplus b)=(x \wedge a)$ $\oplus(x \wedge b)$ for all $x \in L$.

(4) The center of the lattice $L(0, a)=(x ; 0 \leqq x \leqq a)$ consists exactly of all $z \wedge a, z$ in the center of $L$.

(5) If for $a, b \in L, a \perp b$, then $a$ and $b$ are strongly perspective if and only if they are perspective.

(6) If for $a, b \in L, a \perp b$ and $a \sim b$, then $L(0, a)$ and $L(0, b)$ are isomorphic.

(7) If for $a, b, c \in L,(a, b, c) \perp$ and $a \sim b, b \sim c$, then $a \sim c$.

Proof. Statements (2), (3), (5), (6), and (7) were first proved for $L$ complemented modular by von Neumann. All are contained in [13, Part I]: (2) is Theorem 5.7, (3) is Theorem 5.1,(5) is Theorem 3.1,(6) is Theorem 3.3 and (7) is Theorem 3.4. Statements (1) and (4) were proved first for $L$ a continuous geometry by von Neumann [13, Theorem 5.9 of Part I and Theorem 1.6 of Part III], and later generalized to $L$ complete complemented modular by Kaplansky $[5, \S 8$; (4) is Theorem 4 and (1) follows easily from Theorem 4 and Lemma 36]. If $L$ is the projection lattice of a von Neumann algebra then (1) through (7) can be proved by standard methods of the theory of these algebras and we omit the details. We demonstrate the failure of (1) through (7) in the general complete orthomodular lattice by means of a single counterexample, whose Hasse diagram is shown in Figure 1. One checks easily that this lattice is orthomodular and that its center consists of just 0 and 1 . We now show that each of the statements (1) through (7) is false for this lattice, which we denote by $L$ in the remainder of the proof. We are indebted to the referee for pointing out to us that this lattice appeared already in a paper by Dilworth published in 1940 [14, p. 21]. In this paper Dilworth already observed that it is orthomodular (in his notation: "closed with respect to relative negation') but not modular.

(1) We claim that $(a, g) P$. To prove this we must show that $a \wedge g=0$ and that for an arbitrary element $x \in L,(a, c, x)$ is a distributive triple. That $a \perp g$ is obvious by inspection, and so in particular we have $a \wedge g=0$. To prove that for any $x(a, c, x)$ is a distributive triple we observe simply that no matter what element we choose for $x$, at least one of the relations $x \geqq a, x \geqq g, x \perp a, x \perp g$ holds. Consequently every element of $L$ commutes with either $a$ or $g$. Since also $a C g$, we conclude by Lemma 5 that $(a, g, x)$ is a distributive triple no matter what element 


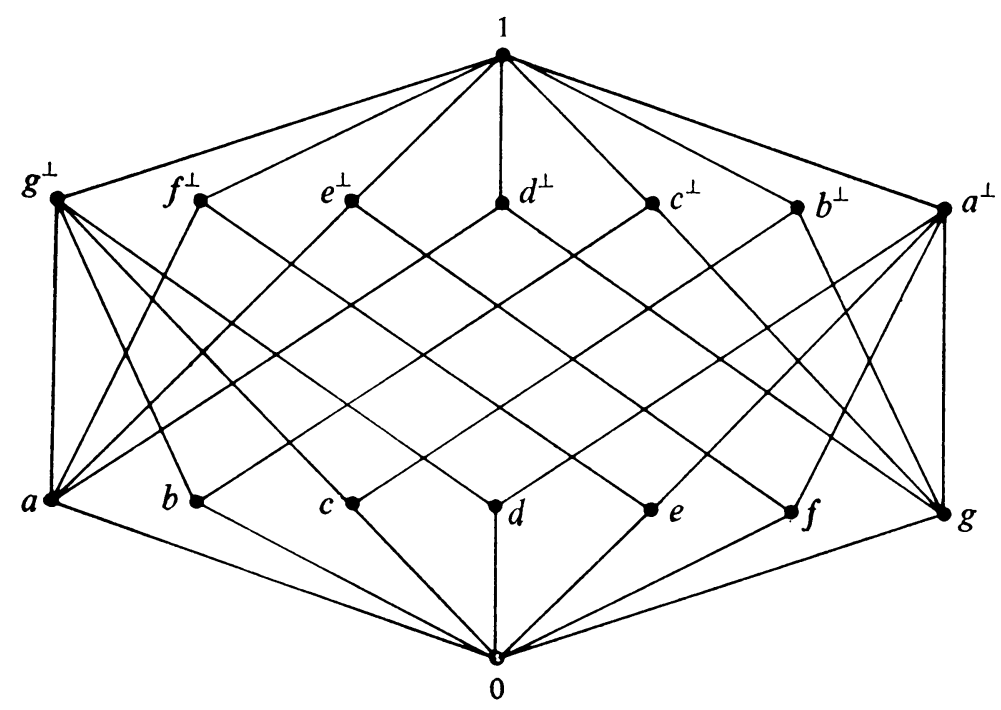

FIGURE 1

is chosen for $x$. This shows that $(a, g) P$. It is easy to see that $g$ is the largest such element, so $g=a^{\nabla}$. But since $a^{\nabla}=g \neq 0,1, a^{\nabla}$ is not central.

(2) Observe that $a$ and $e$ satisfy the condition: $a_{1} \leqq a, e_{1} \leqq e, a_{1} \sim e_{1}$ imply $a_{1}=e_{1}=0$. This follows immediately from the fact that they are atoms, and are not themselves strongly perspective. (Since $a$ and $e$ are the only nonzero elements in $a \vee e=f^{\perp}$, there is no element to effect the strong perspectivity.) But $(a, e) P$ does not hold, since $(a, e, c)$ is not a distributive triple.

(3) One verifies directly that the second condition of (3) is satisfied by the pair $a, e$ but as observed in (2), $(a, e) P$ does not hold.

(4) $L\left(0, f^{\perp}\right)$ is a four element Boolean lattice which has consequently a four element center. But the set $\left(z \wedge f^{\perp}, z\right.$ in the center of $\left.L\right)$ has but two elements, since the center of $L$ consists of just 0 and 1 .

(5) The elements $a$ and $e$ are orthogonal, and as shown in (2) are not strongly perspective. However they are perspective via the element $b^{\perp}$.

(6) The elements $b$ and $b^{\perp}$ are strongly perspective via $e$. But $L(0, b)$ has two elements and $L\left(0, b^{\perp}\right)$ has four so they are not isomorphic.

(7) The element $a$ is strongly perspective to $d$ via $b$ and $d$ is strongly perspective to $g$ via $f$. Also $(a, d, g) \perp$. But $a$ is not strongly perspective to $g$, since $a$ and $g$ are the only nonzero elements contained in their span, $d^{\perp}$. This completes the proof of Theorem 4 .

\section{BIBLIOGRAPHY}

1. A. Brown, On the absolute equivalence of projections, Bul. Inst. Politehn. Iassi (8) 4 (1958), $5-6$.

2. D. J. Foulis, A note on orthomodular lattices, Portugal. Math. 21 (1962), 65-72. 
3. P. R. Halmos, Introduction to Hilbert space, 2nd ed., Chelsea, New York, 1957.

4. S. S. Holland, Jr., A Radon-Nikodym theorem in dimension lattices, Trans. Amer. Math. Soc. 108 (1963), 66-87.

5. I. Kaplansky, Any orthocomplemented complete modular lattice is a continuous geometry, Ann. of Math. (2) 61 (1955), 524-541.

6. - Rings of operators, Notes, Univ. of Chicago, Chicago Ill., 1955 (unpublished).

7. L. H. Loomis, The lattice theoretic background of the dimension theory of operator algebras, Mem. Amer. Math. Soc. No. 18 (1955), 36 pp.

8. G. W. Mackey, Lecture notes on the mathematical foundations of quantum mechanics, Harvard Univ., Cambridge, Mass., 1960 (unpublished).

9. F. Maeda, Kontinuierliche Geometrien, Springer, Berlin, 1958.

10. - Decomposition of general lattices into direct summands of types I, II and III, J. Sci. Hiroshima Univ. Ser. A 23 (1959), 151-170.

11. S. Maeda, Dimension functions on certain general lattices, J. Sci. Hiroshima Univ. Ser. A 19 (1955), 211-237.

12. M. Nakamura, The permutability in a certain orthocomplemented lattice, Ködai Math. Sem. Rep. 9 (1957), 158-160.

13. J. von Neumann, Continuous geometry, Princeton Univ. Press, Princeton, N. J., 1960.

14. R. P. Dilworth, On complemented lattices, Tôhoku Math. J. 47 (1940), 18-23.

15. G. W. Mackey, On infinite-dimensional linear spaces, Trans. Amer. Math. Soc. 57 (1945), 155-207.

\section{Boston College,}

Chestnut Hill, Massachusetts 